\title{
Consumo de riesgo de drogas utilizando la herramienta ASSIST modificado en estudiantes, docentes y personal administrativo de una universidad pública.
}

\author{
Santiago Piedra ${ }^{1}$, Alberto Narváez ${ }^{2}$, Patricio Jácome ${ }^{3}$, Raúl Terán ${ }^{4}$, \\ Dimitri Barreto ${ }^{5}$, Romel Aguirre ${ }^{2}$, Hugo Noboa ${ }^{6}$
}

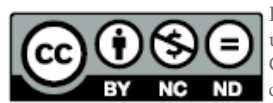

artículo está bajo na licencia de Creative Commons de tipo Reconocimiento - No comercial OPEN ACCESS - Sin obras derivadas 4.0 International

1. Médico general - Hospital de Especialidades Carlos Andrade Marín (HECAM)

2. Facultad de Ciencias Médicas - Universidad Central del Ecuador

3. Facultad de Ciencias Psicológicas - Universidad

Central del Ecuador.

4. Observatorio de Salud, Dirección de Vinculación con la Sociedad.

5. Dirección de Comunicación y Cultura - Coordinación Rectorado de la Universidad Central del Ecuador. 6. Dirección de Bienestar Universitario - Red Ecuatoriana de Universidades Promotoras de la Salud y el Bienestar.

ORCID ID:

Santiago Piedra

https:// orcid.org/0000-0003-3003-065X

Alberto Narvaez

https:/ / orcid.org/0000-0002-5056-6315

Patricio Jacome

https:// orcid.org/0000-0001-7303-6643

Received: 03 - Oct - 2019

Accepted: 27 - Dec - 2019

Publish: 31 - Dec - 2019

Corresponding author: Santiago Piedra

E-mail: santpiedra@outlook.com

Authorship: All authors conceptualized an designed the data, collected the data, analyzed and interpreted the data, drafted the article, and critically revised the article.

Financial disclosure: The authors have no financial relationships relevant to this article to disclose.

\section{Resumen}

El consumo de drogas es una importante causa de morbimortalidad a nivel mundial y su impacto genera gastos sanitarios, económicos y sociales importantes. Las mayores tasas de consumo de drogas se observan en la población entre 18 y 25 años en donde una proporción importante son estudiantes universitarios. El objetivo de este estudio fue calcular la prevalencia del consumo de riesgo de sustancias entre estudiantes, profesores y trabajadores de una universidad pública del Ecuador. Además, se realizó el cálculo de la consistencia interna (confiabilidad) del cuestionario ASSIST modificado.

Pacientes y Métodos: Se realizó un estudio analítico transversal, se aplicó la prueba de Alcohol, Smoking and Substance Involvement Screening Test (ASSIST) en dos grupos poblacionales (estudiantes y trabajadores docentes). La consistencia interna de cada subescala del cuestionario fue evaluada con alfa $(\alpha)$ de Cronbach. Para estimar la prevalencia del consumo de riesgo se utilizó porcentajes con intervalos de confianza (IC) del 95\%.

Resultados: 4958 estudiantes y 425 profesores, trabajadores y empleados respondieron el cuestionario ASSIST. El cálculo de la consistencia interna (confiabilidad) del instrumento, demostró un $\alpha$ de Cronbach satisfactorio (>0.7) para consumo de: tabaco, alcohol y marihuana. La prevalencia del consumo de riesgo alto para alcohol (5,3\% para estudiantes y $4,9 \%$ para trabajadores y docentes), tabaco y cannabis fue elevada en los grupos estudiados. Además, el porcentaje de la población con consumo de riesgo moderado también fue significativo.

Conclusión: En virtud de las elevadas tasas de consumo de riesgo alto y moderado para el consumo de drogas, es necesario la implementación de un programa Integral de promoción, prevención, tratamiento y rehabilitación dirigido a estudiantes universitarios. La prueba ASSIST modificada mostró una consistencia interna satisfactoria en la evaluación del consumo de riesgo de substancias.

Palabras clave: tamizaje, ASSIST, consumo de riesgo, consistencia interna

Citation: Piedra S, Narváez A, Jácome

P, Terán R, Barreto D, Aguirre R, et al.

Consumo de riesgo de drogas utilizando

la herramienta ASSIST modificado

en estudiantes, docentes y personal

administrativo de una universidad pública

Rev Med Vozandes. 2019; 30 (2): 19 - 25 
Keywords: substancerelated disorder, screening, psychometrics, ASSIST

\section{Abstract}

\section{Drug risk use using the ASSIST modified in students, teachers and administrative staff of a public university.}

Drug use is a major cause of global morbidity and its impact generates significant health, economic and social expenditures. The highest rates of drug use are seen in the population between the ages of 18 and 25, where a significant proportion are university students. The objective of this study was to calculate the prevalence of substance risk consumption among students, professors and workers at a public university in Ecuador. In addition, the calculation of the internal consistency (reliability) of the modified ASSIST questionnaire was performed.

Patients and Methods: A cross-analytical study was conducted, the Alcohol, Tobacco and Substances Consumption Detection (ASSIST) test was applied in two population groups (students and workers - teachers). The internal consistency of each subscale of the questionnaire was evaluated with Cronbach's alpha. Percentages with $95 \% \mathrm{Cl}$ were used to estimate the prevalence of risk consumption.

Results: 4958 students and 425 teachers, workers and employees responded to the ASSIST questionnaire. The calculation of the internal consistency (reliability) of the instrument, proved Cronbach satisfactory $(>0.7)$ for consumption of tobacco, alcohol and marijuana. The prevalence of high-risk alcohol use $15.3 \%$ for students and $4.9 \%$ for workers and teachers), tobacco and cannabis were high in the groups studied. In addition, the percentage of the population with moderate risk consumption was also significant.

Conclusions: Under the high rates of high and moderate risk use for drug use, it is necessary to implement a comprehensive program of promotion, prevention, treatment and rehabilitation aimed at university students. The modified ASSIST test showed satisfactory internal consistency in the assessment of substance risk consumption.

\section{Introducción}

El consumo de drogas es una importante causa de morbimortalidad a nivel mundial. De la misma forma el Burden económico de la enfermedad genera gastos significativos a los sistemas de salud.

La tasa de mortalidad atribuible al consumo de alcohol y otras drogas ilícitas es de aproximadamente 39 por cada 100,000 habitantes a nivel mundial (90\% por alcohol). Además, es responsable por la pérdida de 13 años de vida ajustados por la discapacidad por cada 1,000 habitantes en el mundo. (1)

La Oficina de las Naciones Unidas Contra la Droga y el Delito en el III Estudio epidemiológico andino sobre consumo de drogas en la población universitaria del Ecuador (2) en 2016 muestra que las mayores tasas de uso de drogas se observan 
en la población joven entre 18 y 25 años de edad $151,5 \%$ en alcohol, $56,4 \%$ en tabaco, $10,3 \%$ en sedantes, $26,9 \%$ en cannabis, $4,4 \%$ en cocaína) donde una proporción importante de ella corresponde a estudiantes universitarios. (3) Los estudios llevados a cabo en los años 2009 y 2012 en el proyecto de apoyo a la reducción de la demanda de drogas ilícitas en la Comunidad Andina, reportados en su tercer estudio epidemiológico en 20172, mostraron que para esta misma población, existe un alarmante uso de marihuana y cocaína. Otro hallazgo notable en estos estudios fue el uso de drogas inhalables entre la población universitaria, particularmente entre las mujeres. ${ }^{(2)}$ Los resultados de estos estudios muestran la necesidad de la implementación de programas integrales para controlar este problema de salud pública.

Por lo tanto, resulta ineludible monitorear la evolución de este problema de salud pública en este grupo de población, con el propósito de conocer la utilidad de los programas de prevención actuales, como también para conocer la tendencia del problema e implementar nuevos programas basados en la evidencia.

Uno de los instrumentos desarrollados para el cribado de personas que consumen drogas y que son susceptibles de recibir intervención es el Alcohol, Smoking and Substance Involvement Screening Test (ASSIST). Fue desarrollado por la OMS para ser aplicado en la atención primaria, donde el consumo perjudicial de drogas suele pasar desapercibido. Fue diseñado para su aplicación en diversas culturas y fue traducido al español por el equipo que lo diseñó. (4) En Chile se realizó el cálculo de la consistencia interna [índice de fiabilidad o alfa $(\alpha)$ de Cronbach] de las sustancias en un centro de tratamiento con resultados satisfactorios para tabaco (0.87), alcohol (0.93), marihuana (0.95), cocaína (0.98), anfetaminas (0.98), inhalantes (0.90), tranquilizantes (0.97), alucinógenos (0.98) y opiáceos (0.98) 1. Este instrumento no ha sido validado previamente en el Ecuador.

La OMS también publicó la base teórica y la eficacia de la intervención oportuna, así como las pautas para optimizar los resultados en la atención primaria en salud en aquellos usuarios cuyo consumo de sustancias los ponen en riesgo. Este cuestionario presenta un enfoque integral, permitiendo detectar e intervenir de forma oportuna. Además, se adapta de forma eficiente a las circunstancias específicas de la atención primaria, mejorando de esta forma la salud de las poblaciones afectadas. ${ }^{(5)}$

El objetivo de este estudio fue estimar la prevalencia del consumo de riesgo de sustancias ilícitas entre estudiantes, profesores y trabajadores de una universidad pública del Ecuador. Además, se realizó el cálculo de la consistencia interna (coeficiente de fiabilidad) del cuestionario ASSIST modificado (prevalencia en el último mes y en los últimos tres meses). Este estudio permitirá implementar un programa integral de promoción de la salud, así como de prevención, tratamiento y rehabilitación de miembros de la comunidad universitaria afectados por el consumo de alcohol, tabaco y otras drogas en una universidad pública.

\section{Metodología}

Se realizó un estudio analítico transversal de base poblacional en una universidad pública de la ciudad de Quito, Ecuador. El estudio tuvo dos grupos de observación: a) estudiantes y b) profesores, empleados y trabajadores. El consentimiento informado fue obtenido de todos los participantes del estudio y fue aprobado por el Comité de Ética de la Universidad.

El cuestionario ASSIT consta de diferentes variables que evalúan el riesgo de consumo y la prevalencia en los tres meses previos a su aplicación. Este estudio evaluó solamente el consumo en el último mes.

La modificación cronológica del cuestionario fue elaborada en base al test ASSIST (6) para Detección de Consumo de Alcohol, Tabaco y Sustancias.

Se digitalizó el cuestionario ASSIST modificado y se lo aplico a los participantes de los dos grupos en el último trimestre del año 2017 (septiembre a diciembre).

El riesgo de consumo se clasificó como: alto, moderado o bajo para cada sustancia en el último mes de acuerdo con las puntuaciones otorgadas a cada una de las dimensiones que el cuestionario evalúa: a) frecuencia con la que se experimenta fuertes deseos de consumir sustancias ilícitas, b) frecuencia con la que se experimentan problemas de salud, sociales, legales o económicos, c) frecuencia con la que el consumo ha llevado a dejar de hacer lo que se esperaba de uno mismo, d) frecuencia con la que un amigo, un familiar o alguien más ha mostrado preocupación por el consumo alguna vez; y si se ha hecho algún intento por controlar, reducir o abandonar el consumo sin haberlo logrado. (4)

A cada respuesta le corresponde una puntuación que, al sumarlas, permiten establecer la categoría de riesgo: bajo (0-3), moderado (4-26) o alto (27 o más puntos). (4)

Una prueba piloto auto administrada fua aplicada a 40 estudiantes del preuniversitario del mismo establecimiento educativo. Con base en esta prueba se hicieron modificaciones en tres preguntas que no entendieron adecuadamente los participantes.

La población de estudio fueron 40.000 estudiantes y 2300 docentes y 1600 servidores administrativos y de servicio aproximadamente. Se excluyeron del estudio las extensiones universitarias y carreras a distancia. Para la promoción y divulgación de la investigación se visitaron las diferentes facultades de la Universidad y se publicaron posts en las diferentes redes sociales.

El número de personas que respondieron la prueba ASSIT fueron: 4958 estudiantes (muestra estimada 
1000) y 425 profesores, trabajadores y empleados (muestra estimada 700). No se alcanzó el tamaño mínimo de muestra estimada en este último grupo.

La prueba ASSIST se administró en línea utilizando la plataforma de la página web de la universidad. Se definieron campos obligatorios de llenado, restricciones de ingreso y saltos condicionados. Previo a la aplicación del instrumento, los estudiantes fueron informados por medio de mensaje de correo de los objetivos y beneficios de su participación. Al final del mensaje se les pidió que registren su deseo de participar o no en la investigación. A las personas que aceptaron participar, se les pidió que llenen el consentimiento informado y la prueba ASSIST modificada.

No se recogió información demográfica (edad, sexo), facultad, carrera, curso para los estudiantes ni la ocupación para los trabajadores ya que por un error informático se eliminaron estas variables, limitando aún más el estudio.

\section{Análisis Estadística}

Se utilizó el programa Epilnfo 7.2 para el análisis univarial. Para variables cualitativas se estimaron porcentajes con intervalos de confianza del 95\%. Para el cálculo del Coeficiente de Fiabilidad (Alfa de Cronbach) de las subescalas del instrumento ASSIST se Utilizó IBM SPSS software package, versión 23.0 for Windows (IBM, Armonk, NY, USA).

\section{Resultados}

Los estudiantes que respondieron el cuestionario ASSIST fueron 4958, mientras que el número de cuestionarios aplicados a profesores, trabajadores y empleados fue de 425. Se calculó el coeficiente de fiabilidad para cada sustancia en cada grupo de participantes (Tabla 1), la fiabilidad fue satisfactoria para tabaco alcohol y cannabis en los dos grupos ( $\alpha$ de Cronbach $>0,70)$. En el grupo de estudiantes los coeficientes $\alpha$ fueron: tabaco 0,8, alcohol 0,75 y marihuana 0,73 y en el grupo de docentes y administrativos: tabaco 0,79, alcohol 0,78 y marihuana 0,74. (Tabla 1).
La mayoría de los participantes de los grupos estudiados presentaron bajo riesgo para las drogas legales. La prevalencia más elevada de consumo de riesgo alto fue para el alcohol $(5,3 \%$ estudiantes y $4,9 \%$ docentes y trabajadores). Le siguen en frecuencia el tabaco $(2,6 \%$ (IC 95\% 2,23,1 ) entre estudiantes; $1,9 \%$ (IC 95\% $0,9 \%-3,8 \%$ ) para docentes y administrativos, y cannabis $10,7 \%$ (IC 95\% 0,5-1,0) en estudiantes y 0,5\% (IC95\% 0,1$1,9)$ en docentes y administrativos). (Tabla 2).

El porcentaje de participantes con riesgo moderado muestra la misma tendencia en los dos grupos de participantes en las sustancias como el tabaco y alcohol $(30 \%$ y $28 \%$ entre estudiantes respectivamente) y $29 \%$ y $24 \%$ (para docentes y administrativos respectivamente) (Tabla 2).

La mayoría de los participantes encuestados presentaron un consumo de riesgo bajo para las drogas ilegales. No obstante, se encontró una elevada prevalencia de consumo de riesgo alto para cannabis en el grupo de estudiantes, $0,7 \%$ (IC95\% 0,5-1,0) y entre docentes y administrativos, 0,5\% (IC 95\% 0,1-1,9) (Tabla 3).

\section{Discusión}

La consistencia interna del cuestionario ASSIST modificado en esta investigación fue satisfactoria para el tamizaje del consumo de riesgo para el alcohol, el tabaco y el cannabis. (7) El cuestionario no ha sido validado previamente en el país (fiabilidad, validez, sensibilidad, factibilidad).

Nuestros resultados coinciden con los de Christoff y colaboradores ${ }^{(8)}$ que encontraron una consistencia interna satisfactoria en las mismas sustancias en un formato digital del instrumento ASSIST. En este estudio además compararon el formato basado en computadora con el formato habitual mediado por el personal de atención primaria en salud. El análisis de satisfacción y factibilidad mostró que ASSIST habitual era más

Tabla 1 - Coeficiente de Fiabilidad (Alfa de Cronbach) de las subescalas del instrumento ASSIST.

\begin{tabular}{|c|c|c|c|c|c|}
\hline Grupo & Subescala & Alfa de Cronbach & Grupo & Subescala & Alfa de Cronbach \\
\hline \multirow{9}{*}{ 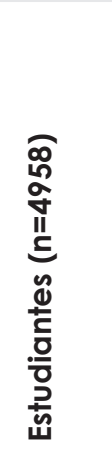 } & Tabaco & 0,80 & \multirow{9}{*}{ 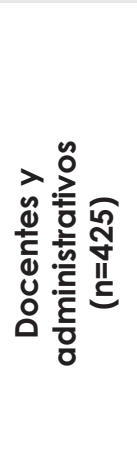 } & Tabaco & 0,79 \\
\hline & Alcohol & 0,75 & & Alcohol & 0,78 \\
\hline & Cannabis & 0,73 & & Cannabis & 0,74 \\
\hline & Cocaína & 0,61 & & Cocaína & 0,59 \\
\hline & Anfetamina & 0,54 & & Anfetamina & 0,49 \\
\hline & Inhalantes & 0,53 & & Inhalantes & 0,60 \\
\hline & Sedantes & 0,61 & & Sedantes & 0,66 \\
\hline & Alucinógenos & 0,56 & & Alucinógenos & 0,51 \\
\hline & Opiáceos & 0,53 & & Opiáceos & 0,51 \\
\hline
\end{tabular}


ARTíCULO ORIGINAL Estudio transversal

Tabla 2 - Nivel de riesgo según la puntuación específica del paciente para cada sustancia. Drogas legales.

\begin{tabular}{|c|c|c|c|c|c|c|c|}
\hline \multirow[b]{2}{*}{ Variable } & & \multicolumn{3}{|c|}{ Estudiantes ( $n=4958$ ) } & \multicolumn{3}{|c|}{ Docentes y administrativos $(n=425)$} \\
\hline & & No & $\%$ & IC $95 \%$ & No & $\%$ & IC $95 \%$ \\
\hline \multirow[t]{3}{*}{ Tabaco } & Riesgo bajo & 3314 & $66,8 \%$ & $(65,5 \%-68,1 \%)$ & 300 & $70,6 \%$ & $(66,0 \%-74,8 \%)$ \\
\hline & Riesgo moderado & 1513 & $30,5 \%$ & $(29,2 \%-31,8 \%)$ & 117 & $27,5 \%$ & $(23,4 \%-32,1 \%)$ \\
\hline & Riesgo alto & 131 & $2,6 \%$ & $(2,2 \%-3,1 \%)$ & 8 & $1,9 \%$ & $(0,9 \%-3,8 \%)$ \\
\hline \multirow[t]{3}{*}{ Alcohol } & Riesgo bajo & 3251 & $65,6 \%$ & $(64,2 \%-66,9 \%)$ & 302 & $71,1 \%$ & $(66,5 \%-75,3 \%)$ \\
\hline & Riesgo moderado & 1446 & $29,2 \%$ & $(27,9 \%-30,5 \%)$ & 102 & $24,0 \%$ & $(20,1 \%-28,4 \%)$ \\
\hline & Riesgo alto & 261 & $5,3 \%$ & $(4,7 \%-5,9 \%)$ & 21 & $4,9 \%$ & $(3,2 \%-7,6 \%)$ \\
\hline \multirow[t]{3}{*}{ Inhalantes } & Riesgo bajo & 4613 & $93,0 \%$ & (92,3\%-93,7\%) & 395 & $92,9 \%$ & $(90,0 \%-95,1 \%)$ \\
\hline & Riesgo moderado & 334 & $6,7 \%$ & $(6,1 \%-7,5 \%)$ & 29 & $6,8 \%$ & $(4,7 \%-9,8 \%)$ \\
\hline & Riesgo alto & 11 & $0,2 \%$ & $(0,1 \%-0,4 \%)$ & 1 & $0,2 \%$ & $(0,0 \%-1,5 \%)$ \\
\hline \multirow[t]{3}{*}{ Sedantes } & Riesgo bajo & 4534 & $91,4 \%$ & $(90,6 \%-92,2 \%)$ & 391 & $92,0 \%$ & $(88,9 \%-94,3 \%)$ \\
\hline & Riesgo moderado & 410 & $8,3 \%$ & $(7,5 \%-9,1 \%)$ & 33 & $7,8 \%$ & $(5,5 \%-10,8 \%)$ \\
\hline & Riesgo alto & 14 & $0,3 \%$ & $(0,2 \%-0,5 \%)$ & 1 & $0,2 \%$ & $(0,0 \%-1,5 \%)$ \\
\hline
\end{tabular}

Tabla 3 - Nivel de riesgo según la puntuación específica del paciente para cada sustancia. Drogas ilegales. Quito, 2018.

\begin{tabular}{|c|c|c|c|c|c|c|c|}
\hline \multirow{2}{*}{ Variable } & & \multicolumn{3}{|c|}{ Estudiantes $(n=4958)$} & \multicolumn{3}{|c|}{ Docentes y administrativos $(n=425)$} \\
\hline & & No & $\%$ & IC $95 \%$ & No & $\%$ & IC $95 \%$ \\
\hline \multirow[t]{3}{*}{ Alucinógenos } & Riesgo bajo & 4573 & $92,2 \%$ & (91,4\%-93,0\%) & 394 & $92,7 \%$ & $(89,7 \%-94,9 \%)$ \\
\hline & Riesgo moderado & 375 & $7,6 \%$ & $(6,9 \%-8,3 \%)$ & 31 & $7,3 \%$ & $(5,1 \%-10,3 \%)$ \\
\hline & Riesgo alto & 10 & $0,2 \%$ & $(0,1 \%-0,4 \%)$ & 0 & $0 \%$ & $(0,0 \%-0,0 \%)$ \\
\hline \multirow[t]{3}{*}{ Opioides } & Riesgo bajo & 4610 & $93,0 \%$ & $(92,2 \%-93,7 \%)$ & 397 & $93,4 \%$ & $(90,5 \%-95,5 \%)$ \\
\hline & Riesgo moderado & 339 & $6,8 \%$ & $(6,2 \%-7,6 \%)$ & 28 & $6,6 \%$ & $(4,5 \%-9,5 \%)$ \\
\hline & Riesgo alto & 9 & $0,2 \%$ & $(0,1 \%-0,4 \%)$ & 0 & $0 \%$ & $(0,0 \%-0,0 \%)$ \\
\hline \multirow[t]{3}{*}{ Cannabis } & Riesgo bajo & 4131 & $83,3 \%$ & $(82,2 \%-84,3 \%)$ & 368 & $86,6 \%$ & $(82,9 \%-89,6 \%)$ \\
\hline & Riesgo moderado & 793 & $16,0 \%$ & $(15,0 \%-17,1 \%)$ & 55 & $12,9 \%$ & $(10,0 \%-16,6 \%)$ \\
\hline & Riesgo alto & 34 & $0,7 \%$ & $(0,5 \%-1,0 \%)$ & 2 & $0,5 \%$ & $(0,1 \%-1,9 \%)$ \\
\hline \multirow[t]{3}{*}{ Cocaína } & Riesgo bajo & 4579 & $92,4 \%$ & (91,6\%-93,1\%) & 394 & $92,7 \%$ & $(89,7 \%-94,9 \%)$ \\
\hline & Riesgo moderado & 364 & $7,3 \%$ & $(6,6 \%-8,1 \%)$ & 31 & $7,3 \%$ & $(5,1 \%-10,3 \%)$ \\
\hline & Riesgo alto & 15 & $0,3 \%$ & $(0,2 \%-0,5 \%)$ & 0 & $0 \%$ & $(0,0 \%-0,0 \%)$ \\
\hline \multirow[t]{3}{*}{ Anfetamina } & Riesgo bajo & 4605 & $92,9 \%$ & $(92,1 \%-93,6 \%)$ & 396 & $93,2 \%$ & $(90,2 \%-95,3 \%)$ \\
\hline & Riesgo moderado & 343 & $6,9 \%$ & $(6,2 \%-7,7 \%)$ & 29 & $6,8 \%$ & $(4,7 \%-9,8 \%)$ \\
\hline & Riesgo alto & 10 & $0,2 \%$ & $(0,1 \%-0,4 \%)$ & 0 & $0 \%$ & $(0,0 \%-0,0 \%)$ \\
\hline
\end{tabular}

Tabla 4 - Estimación de población a Intervenir según Intervalos de confianza del 95\%.

\begin{tabular}{|c|c|c|c|}
\hline & & Estudiantes ( $n=4958$ ) & Docentes y administrativos $(n=425)$ \\
\hline Variable & & Límite inferior IC $95 \%$ No. Personas & Límite inferior IC95\% No. Personas \\
\hline \multirow[t]{2}{*}{ Tabaco } & Riesgo moderado & 11680 & 913 \\
\hline & Riesgo alto & 880 & 35 \\
\hline \multirow[t]{2}{*}{ Alcohol } & Riesgo moderado & 11160 & 784 \\
\hline & Riesgo alto & 1880 & 125 \\
\hline \multirow[t]{2}{*}{ Cannabis } & Riesgo moderado & 6000 & 390 \\
\hline & Riesgo alto & 200 & 4 \\
\hline \multirow[t]{2}{*}{ Cocaína } & Riesgo moderado & 2640 & 199 \\
\hline & Riesgo alto & 80 & 0 \\
\hline
\end{tabular}


fácil de entender, pero los dos formatos se consideraron similares al considerar la aceptabilidad, la facilidad de respuesta y el grado de intimidación. La conclusión de este estudio fue que los dos formatos son aceptables, los puntajes son comparables y se pueden usar indistintamente. ${ }^{\left(\mathbf{8}^{\prime 9}\right)}$ En el presente estudio se utilizó el formato digital en virtud de los resultados de Christoff y colaboradores ${ }^{(8)}$.

No existen estudios que reporten los riesgos asociados al consumo de sustancias en el Ecuador.

El Tercer Estudio Epidemiológico Andino sobre consumo de drogas en la población universitaria realizado en el año de 2009, 2012 y 2016, reportó que el 51,5\% de los estudiantes consumió alcohol al menos una vez durante los últimos 30 días, de los cuales el 58,1\% fueron hombres y el $45,5 \%$ mujeres. (2) Aunque este estudio aplicó un cuestionario diferente al ASSIST, sus resultados no son tan útiles para establecer y evaluar el resultado de futuras intervenciones que se plantean realizar en la universidad donde se efectuó este plan piloto. El presente estudio constituye por tanto la línea de base para el programa de prevención sobre farmacodependencia e intervenciones breves, propuesto por la OMS, para disminuir el consumo de riesgo de estas sustancias. ${ }^{(4)}$

Este estudio además encontró una prevalencia significativa de consumo de riesgo alto para el alcohol en el grupo de estudiantes y en el grupo de docentes y administrativos. Las sustancias con mayores frecuencias de riesgo alto, después del alcohol, fueron el tabaco y el cannabis. Se encontraron también altas frecuencias de consumo de riesgo moderado para alcohol y tabaco en aproximadamente $30 \%$ de los encuestados, $16 \%$ para cannabis y $7 \%$ para el resto de las drogas. Por las altas tasas de riesgo moderado, esta población merece igual importancia que las personas con riesgo alto por la probabilidad de entrar en la categoría de riesgo alto sin intervención.

Estudios en los que utilizaron el cuestionario ASSIST reportan resultados que discrepan de los del presente estudio. LópezRodríguez y colaboradores ${ }^{(3)}$ en 2018 reportaron prevalencias diferentes y patrones de consumo relacionados con el género en atención primaria en España con un cribado de riesgo moderado/alto de tabaco $(41,7 \%)$, alcohol $(15,4 \%)$, los sedantes o los hipnóticos (13,7\%), el cannabis $(5,7 \%)$. Hombres consumían más alcohol y cannabis; mientras que las mujeres tenían un mayor consumo de sedantes/hipnóticos. (3) En Chile (10), los resultados de línea de base de un ensayo clínico aleatorizado reportaron valores superiores de riesgo moderado a los del presente estudio; aproximadamente 52\% de los participantes tenían un consumo de riesgo moderado para el alcohol, $49 \%$ para cannabis y $19 \%$ para cocaína. Algunos participantes tenían un riesgo moderado para más de una sustancia: $13 \%$ para el alcohol y el cannabis, $7 \%$ para el alcohol y la cocaína y $7 \%$ para el cannabis y cocaína. ${ }^{(10)}$

Los porcentajes de consumo de riesgo alto para tabaco en ambos grupos $10,9 \%$ el valor más bajo en el grupo de docentes y administrativos) y de riesgo moderado, muestran la necesidad de un programa integral, considerando el tamaño de la población estudiada. Como se muestra en la Tabla 3, la estimación con base a los limites inferiores de los IC95\% de las cuatro sustancias con más alta frecuencia, evidencian el número elevado de personas que necesitan intervención tanto en estudiantes como en docentes y trabajadores.
Todos los estudios que reportan prevalencias de riesgos derivados de consumo de sustancias sugieren que los estudios futuros se centren en la aplicación de intervenciones breves y trabajen en equipos multidisciplinarios promoviendo cambios en el comportamiento o incluso el conocimiento y adhesión a la comunidad. ${ }^{(11)}$

La OMS elaboró un manual dentro del marco de trabajo del proyecto ASSIST, en donde se presenta la base teórica y la evidencia de la eficacia de la intervención breve. Este manual ayuda a los trabajadores de la atención primaria de salud a proporcionar una intervención rápida, oportuna, simple y eficaz basada en evidencia para usuarios cuyo consumo de sustancias los ponen en riesgo. ${ }^{(5)}$

La técnica de intervención breve se centra en modificar la conducta de los usuarios con relación a la sustancia más frecuentemente usada o la que les causa la mayoría de los problemas (la identificada por el usuario o la que obtuvo la puntuación más alta del ASSIST). La intervención breve vinculada a ASSIST se basa en las técnicas de FRAMES: Feedback (Retroalimentación), Responsibility (Responsabilidad), Advice (Consejo), Menu of Options (Opciones de cambio), Empathy (Empatía) y Self-efficacy (Autoeficacia) y entrevista motivacional. Dura alrededor de tres a 15 minutos y pueden utilizarse los mismos principios en intervenciones más extensas o periódicas si el tiempo lo permite. Consta de 10 pasos principales sugeridos (o los cinco primeros pasos para una intervención más corta de tres minutos). ${ }^{(5)}$

En una revisión realizada por saitz y colaboradores ${ }^{(6)}$, mostró que varios ensayos aleatorios han probado la eficacia de la intervención breve para el uso de drogas identificadas mediante la detección en entornos de salud general (es decir, en personas que no buscan ayuda para su uso de drogas). Pruebas sustanciales ahora sugieren que la eficacia es limitada o inexistente. Las razones probablemente incluyen un rango de gravedad real y percibida (o falta de gravedad), consumo concomitante poco saludable de alcohol, comorbilidades mentales y los diferentes tipos de consumo y poli consumo de drogas. Si bien la intervención breve puede tener cierta eficacia para los usuarios de drogas que buscan ayuda, el modelo de intervención breve que tiene efectos en entornos de atención primaria sobre el consumo riesgoso de alcohol puede no ser eficaz para el uso de otras drogas. ${ }^{(12)}$

En $2017^{(10)}$, se realizó un ensayo clínico aleatorizado en Chile en donde reportaron la efectividad de la intervención breve (BI) asociada con el ASSIST para el uso de alcohol y drogas ilícitas como parte de un programa de detección sistemática implementado en servicios de atención primaria. En este estudio se identificaron y aleatorizaron 
806 participantes que no buscaron tratamiento, es decir en un entorno de salud general y se comparó la intervención breve con un folleto informativo sobre el riesgo asociado con el uso de sustancias. Los autores concluyeron que no está claro si una intervención breve asociada con el ASSIST es más efectiva que un folleto informativo para reducir el consumo de alcohol y sustancias ilícitas en usuarios de atención primaria que no buscan tratamiento y que tienen un riesgo moderado. ${ }^{(10)}$

Sin embargo, Assanangkornchai y colaboradores (13), en 2015 asignaron al azar pacientes tailandeses en "riesgo moderado" para recibir Intervención Breve ligada a ASSIST $(n=120)$ o consejería sola $(n=116)$. Los resultados fueron cambios en las puntuaciones de participación de sustancias específicas de ASSIST, puntuaciones de participación de sustancias totales de ASSIST y proporciones de pacientes cuyas puntuaciones a los tres y seis meses habían disminuido de "riesgo moderado" a la categoría de "bajo riesgo". Los porcentajes de pacientes convertidos a la categoría de "bajo riesgo" fueron $36.7 \%$ y $38.8 \%$ a los 3 meses y $53.3 \%$ y $53.4 \%$ a los 6 meses para los grupos de Intervención breve y consejería simple, respectivamente. En conclusión, en la atención primaria, administrar ASSIST y decirles a los pacientes su puntuación, seguida de una breve intervención formal o un simple consejo, son igualmente efectivos para disminuir el consumo de sustancias hasta por seis meses. ${ }^{13)}$

Los resultados de este estudio muestran la necesidad de implementación de un programa Integral de promoción de la salud, así como de prevención, tratamiento y rehabilitación de miembros de la comunidad universitaria afectados por el consumo de alcohol, tabaco y otras drogas. El programa de promoción debe incluir la combinación de técnicas de consejería cara a cara por pares y por medios virtuales.

Existen herramientas basadas en evidencia científica para evitar el aumento de la prevalencia de riesgos en esta población vulnerable que están disponibles y validadas. El estudio de Da Silva y colaboradores (11), señala la necesidad de realizar investigaciones que exploren la indicación de intervención, de acuerdo con su puntuación, exactamente porque se trata de un objeto de estudio complejo que requiere atención y prácticas asertivas en la salud de la comunidad (11). Se recomienda desarrollar un estudio de intervención que tome en cuenta estas recomendaciones, así como la aplicación simultánea de instrumentos validados previamente para la elaboración de estudios completos de validación del instrumento en el Ecuador.

En virtud de la no inclusión de variables sociodemográficas no se pudo conocer si la muestra estudiada es representativa de la población universitaria. Sin embargo, la alta precisión alcanzada en estudiantes por el tamaño de muestra es posible que las prevalencias estén cercanas a la realidad.

Otra limitación de nuestro estudio seria la evaluación apenas del consumo de riesgo de alcohol, tabaco y cocaína, este ¡sesgo de inclusión! disminuye la fiabilidad de este instrumento (modificado al propuesto por la OMS).

\section{Conclusión}

El cálculo de la consistencia interna nos permite confiar en los puntajes de las sustancias con valores satisfactorios lo que limita los resultados de las sustancias con fiabilidad baja y en el grupo que no se alcanzó la muestra estimada (trabajadores), por tanto, se torna necesario la implementación de un programa Integral de promoción, prevención, tratamiento y rehabilitación dirigido a estudiantes universitarios. Además, los valores de consistencia interna el cuestionario ASSIST modificado puede ser usado para evaluar el consumo de riesgo de substancias.

\section{Referencias}

1. Soto-Brandt G, Huidobro RP, Artigas DH, et al. Evidencia de validez en Chile del alcohol, smoking and substance involvement screening test (ASSIST). Adicciones. 2014;26(4):291302. doi:10.20882/adicciones.27.

2. Oficina de las Naciones Unidas contra la Droga y el Delito [UNODC]; Comisión Nacional para el Desarrollo y Vida sin Drogas [DEVIDA] III Estudio epidemiológico andino sobre alteraciones en las funciones ejecutivas y consumo de drogas en la población universitaria de Ecuador, 2016. 2017;1:47

3. López-Rodríguez JA, Rigabert A, Gómez Llano $M N$, Rubio $G$. Drug using risks screening in primary care patients using the ASSIST test: Cross sectional study. Aten Primaria. 2018; (xx). doi:10.1016/j.aprim.2017.03.021.

4. Pérez Moreno P, Calzada Álvarez N, Rovira Guardiola J, Torrico Linares E. Estructura factorial del test ASSIST: aplicación del análisis factorial exploratorio y confirmatorio. Trastor Adict. 2012;14(2):44-49. doi:10.1016/\$1575 0973(12)70043-0.

5. Organización Mundial de la Salud. Intervención Breve Vinculada a ASSIST Para El Consumo
Problemático de Sustancias - Manual Para Uso En La Atención Primaria.; 2011 . www.paho.org.

6. Sainz MT, Rosete-Mohedano MG, Rey GN, V? lez NAM, Garc? a SC, Cisneros DP. Validity and reliability of the alcohol, smoking, and substance involvement screening test (ASSUbstance involvement screening test (ASbilidad de la prueba de deteccien de consumo de alcohol, tabaco y sustancias (ASSIST) en estudiantes universitario. Adicciones. 2016;28(1):19-27.

7. Bland JM Altman DG Cronbach's Alpha. $\mathrm{Br}$ Med J. 1997:314:572. doi:https://doi. org/10.1136/bmj.314.7080.572.

8. Christoff AO, Barreto HGA, Boerngen-Lacerda R. Development of a Computer-Based Forma for the Alcohol, Smoking, and Substance Involvement Screening Test (ASSIST) With University Students. Subst Use Misuse. 2016:51(9):12071217. doi:10.3109/10826084.2016.1161053.

9. Wolff N, Shi J. Screening for substance use disorder among incarcerated men with the alcohol, smoking, substance involvement screening test (ASSIST): A comparative analysis of computer-administered and interviewer- administered modalities. J Subst Abuse Treat. 2015;53:22-32. doi:10.1016/j.jsat.2015.01.006.

10. Poblete F, Barticevic NA, Zuzulich MS, et al. A randomized controlled trial of a brief intervention for alcohol and drugs linked to the Alcohol, Smoking and Substance Involvement Screening Test (ASSIST) in primary health care in Chile. Addiction. 2017;112(8):1462-1469. doi: 10.1111 /add.13808.

11. da Silva AC, Lucchese R, Vargas Silva L, Benício PR, Ivânia V. Application of the Alcohol Smoking and Substance Involvement Screening Test (ASSIST) instrument: an integrative review. Rev Gaúcha Enferm. 2016;37(1):1-10. doi:10.1590/1983.

12. Saitz R. Screening and brief intervention for unhealthy drug use: Little or no efficacy. Front Psychiatry. 2014;5(AUG):1-6. doi:10.3389/fpsyt.2014.00121.

13. Assanangkornchai S, Nima P, MCNeil EB, Edwards JG. Comparative trial of the WHO ASSIST-linked brief intervention and simple advice for substance abuse in primary care. Asian Psychiatr. 2015;18(15):75-80. doi:10.1016/j. ajp.2015.09.003. 